\title{
Diseño e implementación de un sistema fotovoltaico híbrido y desarrollo de su regulador de carga aplicando instrumentación virtual
}

\author{
Johan Sebastián Patiño Abella ${ }^{\star}$, Juan Sebastian Tello Reyes ${ }^{\star \star}$, Johann \\ Alexander Hernández Mora ${ }^{\star \star \star}$ \\ Departamento de Ingeniería Eléctrica, Universidad Distrital Francisco José de Caldas, \\ Bogotá, Colombia
}

FeCha de ENTRega: Marzo 14 DE 2012

Fecha de evaluación: Abril 4 de 2012

FECHA DE APROBACIÓN: MAYO 3 DE 2012

\begin{abstract}
Resumen El presente artículo expone las características y las condiciones operativas bajo las cuales funciona un sistema fotovoltaico híbrido desarrollado para ser la fuente de energía de respaldo para diferentes equipos AC que sean dispuestos como cargas críticas a las cuales se les deba garantizar un suministro de energía constante. El sistema se compone de los elementos básicos de un Sistema Fotovoltaico Autónomo común (módulos y baterías), una caja de maniobras que permitirá la interconexión entre los diferentes elementos del sistema, y de una UPS que funciona como interfaz para la alimentación de las cargas críticas, ya sea que esta provenga de la red de suministro eléctrico o del generador fotovoltaico. Para el control de carga del sistema, se implementó un regulador de desarrollado con instrumentación, el cual realiza un monitoreo constante de las tensiones del sistema para emitir las señales de activación de la caja de maniobra, y realizar un registro de las operaciones efectuadas.
\end{abstract}

Palabras Clave: sistemas fotovoltaicos híbridos, instrumentación virtual, reguladores de carga, LabVIEW, dimensionamiento de sistemas fotovoltaicos.

\begin{abstract}
This article presents operative characteristics and conditions under which a hybrid photovoltaic system developed to be the backup electrical source of AC equipment used as critical charges that should be guaranteed to have a constant energy supply functions. The system is made up of the basic elements of a common Autonomous Photovoltaic System (modules and batteries), a box of maneuvers that will enable the interconnection among different elements of the system, and a UPS that acts as an interface to feed critical charges that come from the electrical supply network or from the photovoltaic generator.
\end{abstract}

\footnotetext{
* jsebastian.patino@gmail.com

$\star \star$ jstello87@gmail.com

$\star \star \star$ jahernandezm@udistrital.edu.co
} 
For controlling the system charge, we implemented a developed regulator with instruments. This regulator constantly monitors the tensions of the system for issuing activation signals and registering the operations that were carried out.

Keywords: hybrid photovoltaic systems, virtual instruments, load regulators, LabVIEW, photovoltaic system design.

\section{Introducción}

La tecnología de la energía fotovoltaica tiene cada vez más acogida en su implementación para la generación distribuida, ya que además de usar una fuente primaria de energía inagotable, posee gran facilidad de instalación y requiere un mantenimiento mínimo. Otra característica de esta tecnología es la facilidad con la que se puede integrar a la red eléctrica, por lo que los lugares en los que se adopte pueden llegar a convertirse en sitios energéticamente autónomos que entregan electricidad a la red en lugar de realizar un consumo de la misma [1].

Las redes de suministro eléctrico están expuestas a la ocurrencia de una gran diversidad de eventos que causan fallas en el sistema, lo que finalmente se ve reflejado en cortes de energía. La energía solar se muestra como una de las posibles viables soluciones a esta problemática, ya que puede funcionar como respaldo si en el sitio afectado por la falla operan equipos a los cuales se les debe garantizar un suministro de energía constante por la importancia de las funciones que desarrollan [2].

Este estudio presenta el desarrollo de un sistema al cual se encuentra asociada una carga con las anteriores características, la cual en adelante llamaremos carga crítica, donde el caso de aplicación corresponde a un sistema de monitoreo de variables ambientales y eléctricas que opera durante las 24 horas del día [3]. La novedad del sistema propuesto se basa en tener una disposición no convencional de sistemas fotovoltaicos, ya que este posee características de sistemas fotovoltaicos autónomos y sistemas fotovoltaicos interconectados, entendiéndose un sistema autónomo como aquel que energiza cargas a partir del uso de baterías y módulos fotovoltaicos; y un sistema interconectado como aquel donde la energía solar generada se orienta hacia un inversor para conectarse a la red de suministro eléctrico en el punto de acople común. Por otra parte, se desarrolló un regulador de carga mediante instrumentación virtual que puede reemplazar los reguladores convencionales dispuestos para sistemas fotovoltaicos, lo que permite adicionar mayores herramientas de adquisición y procesamiento de señales del sistema, realizar cambios de los parámetros operativos del mismo según los equipos utilizados, realizar operaciones de conexión y desconexión automática según las señales captadas, y realizar registros de eventos en forma cronológica para evaluar el desempeño del sistema.

\section{Descripción del sistema desarrollado}

Debido a que la carga crítica del sistema opera ininterrumpidamente durante todo el día, fue necesario desarrollar un sistema que garantizara el suministro de 
energía con capacidad de mantener operando el equipo durante un largo período de tiempo en caso de que ocurran cortes del fluido eléctrico. El aporte realizado por el sistema desarrollado consiste en usar un sistema "híbrido" constituido por una fuente de energía convencional (red) y una no convencional (sistema fotovoltaico), donde esta última funciona como respaldo ante cualquier problema de discontinuidad del suministro eléctrico por fallas en la red.

El propósito del sistema planteado responde al momento en que se presente ausencia de tensión en la red de suministro eléctrico o ésta tenga un valor de tensión por debajo de los límites regulatorios, un arreglo de módulos fotovoltaicos y baterías se conecte en paralelo a la batería contenida en una UPS con la que alimenta la carga crítica, lo que permite reorientar la energía de este arreglo hacia la UPS para aumentar el monto de energía que se puede entregar durante el espacio de tiempo que dure la falla. Debido a que el arreglo de módulos fotovoltaicos y baterías alimenta la carga solo por el espacio de tiempo que dura la falla, la cantidad de equipos necesarios para realizar el suministro de energía es mínima, además si la energía demandada es menor que la energía que el arreglo está en capacidad de entregar, los excedentes de energía se destinan a recargar las baterías y de esta forma incrementar el respaldo energético realizado.

\section{Composición del sistema híbrido}

Como se menciona anteriormente, el sistema desarrollado se compone básicamente de los módulos fotovoltaicos y baterías que se conectan a la UPS, aunque se pueden adicionar al sistema diversas cargas de tipo DC, que sean alimentadas por el sistema fotovoltaico, es importante que la tensión de la red mantenga el valor adecuado. La figura 1 muestra un diagrama de bloques del sistema híbrido desarrollado para suministrar la demanda de energía que suponen las cargas dispuestas para el mismo:

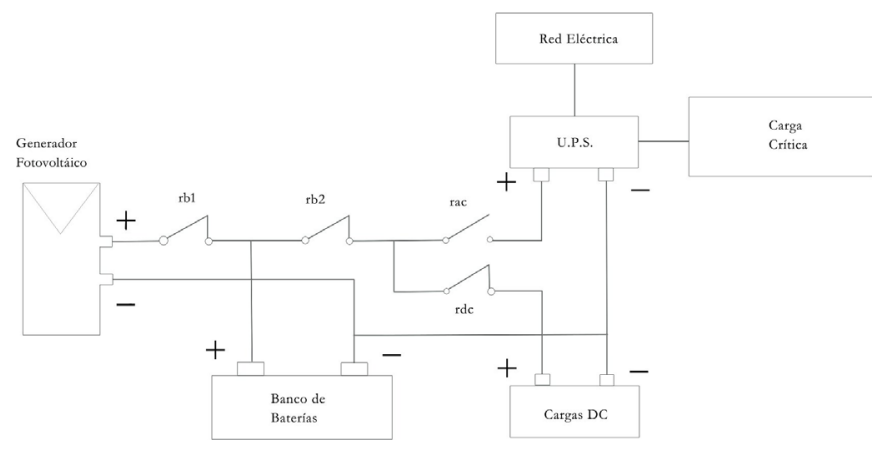

Figura 1. Esquema del sistema fotovoltaico híbrido. 
Como se ve en el esquema, existen dos contactos que interconectan los elementos del sistema para adoptar diferentes disposiciones según se presente el adecuado nivel de tensión de la red y en bornes de las baterías, protegiendo estas últimas contra sobrecargas o descargas profundas que atenten contra la integridad de la batería. La activación de los relés y medición de los niveles de tensión presentes en el sistema se lleva a cabo mediante el uso un control que opera como regulador de carga a partir de instrumentación virtual, el cual captura las señales de tensión y procesa las mismas, para posteriormente emitir las señales digitales para la activación de los relés a los que se asocian los contactos antes mencionados, generando dichas señales según la idoneidad del nivel de tensión leído en la red y en las baterías. A continuación se definen las funciones de los contactos presentes en el sistema híbrido:

- RB1: este relé permite la conexión y desconexión del arreglo de módulos fotovoltaicos al sistema para controlar la sobrecarga de las baterías.

- RB2: este relé permite la conexión y desconexión de las cargas asociadas al sistema para el control de descarga de las baterías.

- RDC: este relé permite la conexión y desconexión de las cargas DC demostrativas en el sistema.

- RAC: este relé permite la conexión de la UPS al arreglo de módulos fotovoltaicos y baterías para la realización del respaldo en la alimentación del equipo de monitoreo.

\section{Modos de operación del sistema híbrido}

Dadas las diferentes situaciones que se pueden presentar durante el funcionamiento del sistema, se hace necesario detallar la forma en la que el sistema afronta cada una de ellas, por lo que se consideraron los siguientes casos:

- Operación normal: el sistema se encuentra en operación normal cuando el nivel de tensión leído de la red es mayor o igual al nivel de tensión mínimo establecido según las normas regulatorias locales, además que la tensión de las baterías se encuentra dentro del rango de valores en los cuales cada fabricante establece que las mismas se encuentran descargadas o sobrecargadas Para esta condición, los relés RB1, RB2 y RDC están cerrados, mientras que RAC está abierto, para que de esta forma los paneles fotovoltaicos y baterías alimenten las cargas DC.

- Condición de falla en suministro de la red: en condición, la tensión en la red de suministro para el equipo de monitoreo está por debajo de la tensión mínima establecida, por lo que el arreglo de módulos y baterías se conecta en paralelo a la UPS para respaldar la misma. En éste estado, los relés RB1, RB2 y RAC se encuentran cerrados, mientras que RDC se encuentra abierto.

- Condición de descarga de baterías: cuando las cargas realizan una alta exigencia de corriente o lo hacen a una tasa moderada, pero por un tiempo prolongado, la tensión de las baterías disminuye hasta el punto en el que se consideran descargadas, según especificaciones establecidas por el fabricante. 
En esta condición el relé RB2, se abre para aislar las cargas del sistema, mientras que RB1 se encuentra cerrado para realizar la carga sobre las baterías a partir de la energía generada por los paneles fotovoltaicos. Aunque se esté energizando la carga crítica (sistema de monitoreo), ésta se desconecta del sistema ya que es preferible cortar el suministro a la carga que causar un daño irreversible a la batería.

- Condición de sobrecarga de las baterías: cuando no hay exigencia de carga o esta es mínima, y el arreglo de módulos fotovoltaicos se encuentra sometido a una alta radiación solar, las baterías tomarán la mayor parte de la corriente foto-generada hasta llegar a un punto en que su carga está a tope. Bajo este principio de operación, los relés RB2 y RDC se encuentran cerrados mientras que RB1 se encontrará abierto, pero si la magnitud del nivel de tensión en la red no es el adecuado, el relé RAC se cierra mientras el relé RDC se abre, por lo que la demanda de carga es atendida exclusivamente por las baterías dado que los paneles no pueden entregar una corriente de magnitud constante.

\section{Dimensionamiento del sistema}

Dado que la luz solar es un recurso cuya intensidad no es constante y que se encuentra disponible solo en una franja horaria del día, el dimensionamiento de un Sistema Fotovoltaico se debe realizar a partir de la energía requerida por las cargas según la potencia consumida y la cantidad de horas de funcionamiento, ya que un dimensionamiento a partir de la potencia supone un suministro de corriente continuo.

\subsection{Metodología de dimensionamiento}

La metodología aplicada para el dimensionamiento del sistema, en una primera instancia, consiste en la definición de la energía total a suministrar, es decir, que se establece la potencia nominal de las cargas y la cantidad de horas del día en las que se encuentra funcionando. La energía que demandan las cargas DC y AC se determinan de la siguiente forma:

$$
\text { Energía }_{D C}=\sum_{i}^{k} P_{i} \times n_{i} \quad[W h]
$$

$i$ : elemento considerado.

$P_{i}$ : potencia nominal del elemento.

$n_{i}$ : número del horas al día que se encuentra en uso.

$$
\text { Energía }_{A C}=\frac{\sum_{i}^{k} P_{i} \times n_{i}}{\text { factor de inversión }} \quad[W h]
$$

$i$ : elemento considerado.

$P_{i}$ : potencia nominal del elemento.

$n_{i}$ : número de horas al día que se encuentra en uso. 
En el caso de disponer de cargas AC, se necesita un inversor para la alimentación de dichas cargas, por lo que el factor de inversión está asociado a la eficiencia de operación de dicho inversor.

A continuación se define la energía que debe suministrar el sistema para atender la demanda de las cargas dispuestas para el sistema.

$$
\text { Energía }_{T O T A L}=\text { Energía }_{A C}+\text { Energía }_{D C} \quad[W h]
$$

$$
\text { Energía total corregida }=\left(\text { Energía }_{\text {TOT AL }}\right) \times(1+F S) \quad[W h]
$$

El término $F S$ corresponde al factor de seguridad, el cual se dispone para realizar un sobre-dimensionamiento en orden dar más confiabilidad al sistema y compensar cualquier tipo de pérdida de energía que se pueda presentar.

Para establecer las condiciones de carga y corriente presentes en el sistema dispuesto, las siguientes variables se encuentran a fin de seleccionar los elementos adecuados que permitirán realizar la instalación del sistema.

Tamaño del generador fotovoltaico. En este apartado se evaluará la cantidad de módulos en paralelo y en serie que conformarán el arreglo dispuesto para realizar la alimentación del sistema.

Cantidad de módulos en serie. Los módulos fotovoltaicos se encuentran en tensiones típicas de $12 \mathrm{Vdc}$ o $24 \mathrm{Vdc}$, por lo que a partir del establecimiento de un voltaje nominal del sistema, se determina la cantidad de módulos que se deben conectar en serie para que el generador fotovoltaico tenga la tensión nominal que se estableció para el sistema.

$$
\text { \# Módulos en serie }=\frac{V_{\text {sistema }}}{V_{\text {nominal del módulo }}}
$$

Cantidad de módulos en paralelo. Para establecer la cantidad de ramas de módulos fotovoltaicos que se deben colocar en paralelo, se determinan las siguientes variables:

- $C i$, Carga equivalente en corriente: en este apartado se evalúa la corriente en A.h que debe suministrar el generador ante las exigencias de carga propuestas en la operación del sistema.

$$
C_{i}=\frac{\text { Energía total corregida }}{\text { V. del sistema }} \quad[A . h]
$$

- Corriente pico del generador: se necesita saber la corriente entregada por el generador cuando éste se expone a la radiación Standard de $1000 \mathrm{~W} / \mathrm{m}^{2}$, debido a que esta corriente corresponde al punto donde el generador entrega la máxima potencia.

$$
\text { I. pico del generador }=\frac{C_{i}}{H S S} \quad[A m p]
$$


El término $H S S$ corresponde a las horas de sol estándar presentes en el sitio de la instalación del sistema.

- Cantidad de módulos en paralelo: dado que la corriente pico del generador es la suma de la corriente suministrada por el conjunto de paneles que conforman el generador fotovoltaico, la cantidad de módulos en paralelo se define de la siguiente forma:

$$
\text { \# Módulos en paralelo }=\frac{\text { I. pico del generador }}{I m \text { del módulo }}
$$

Im es la corriente en el punto de máxima potencia del módulo.

Banco de baterías. El propósito del establecimiento de dichas variables es conocer la cantidad de días de autonomía que se pueden tener bajo las condiciones de carga a las que se somete el sistema.

Cantidad de Baterías en serie. Para establecer esta cantidad, se sigue el mismo racionamiento expuesto para determinar la cantidad de módulos fotovoltaicos en serie.

$$
\text { \# Baterías en serie }=\frac{\mathrm{V} \text {. sistema }}{\mathrm{V} . \text { nominal de la batería }}
$$

Cantidad de baterías en paralelo. Esta cantidad permite encontrar la cantidad de baterías que lograrían una capacidad de corriente suficiente para atender las cargas del sistema. Para este propósito se encuentran las siguientes variables:

- Capacidad nominal del banco de baterías: según los días de autonomía que se dispongan para el sistema, se encuentra la capacidad que debe tener el banco de baterías para atender la carga de acuerdo a los días de autonomía establecidos.

$$
\text { Capacidad nominal del banco }=C_{i} \times \text { días de autonomía }[A . h]
$$

$C_{i}$ es la carga equivalente en corriente que debe entregar el generador fotovoltaico.

- Capacidad corregida del banco: ya que las baterías no se pueden descargar totalmente, la profundidad de descarga determinará la verdadera capacidad que se debe poseer para atender los requerimientos de carga.

$$
\text { Capacidad corregida del banco }=\frac{\text { Capacidad nominal del banco }}{P . d}
$$

P.d: Profundidad de descarga de la batería.

- Cantidad de baterías en paralelo: esta cantidad se define a partir de la relación entre la capacidad corregida del banco de baterías y la capacidad que posee una sola batería.

$$
\text { \# Baterías en paraleo }=\frac{\text { Capacidad corregida del banco }}{\text { Capacidad nominal de la batería }}
$$




\subsection{Dimensionamiento del sistema híbrido para suministro de energía al equipo de monitoreo dispuesto como carga crítica}

Aplicando esta metodología de dimensionamiento, se implementó un sistema fotovoltaico híbrido en el Laboratorio de Materiales Semiconductores y Energía Solar de la Universidad Nacional de Colombia, donde la carga crítica establecida corresponde a un computador y un equipo de adquisición de datos que monitorean un sistema fotovoltaico conectado a la red; así como una nevera de alimentación DC que contiene materiales químicos que son primordiales para las actividades del laboratorio. Las cargas consideradas para el sistema son las siguientes:

\section{Cargas DC:}

- Nevera: debido a que el consumo de potencia de este elemento no es constante durante el funcionamiento del mismo, se realizó un estudio del consumo de energía del elemento, y se encontró que el consumo de energía es de 208 Wh/día. Este refrigerador opera a una tensión de alimentación de $12 \mathrm{~V}$.

\section{Cargas AC:}

- Computador: tensión de alimentación de $120 \mathrm{~V}$ y consumo de $160 \mathrm{~W}$.

- Equipo de adquisición de datos: tensión de alimentación de 120 V y consumo de $60 \mathrm{~W}$.

- Pantalla: tensión de alimentación de $120 \mathrm{~V}$ y consumo de $22 \mathrm{~W}$.

En este caso en particular, al aplicar la metodología antes mencionada no se busca encontrar la cantidad de módulos fotovoltaicos y baterías que son necesarios para atender la carga que representan los elementos ya mencionadas, sino que se busca encontrar la cantidad de horas de operación que pueden tener dichos elementos durante el día para que los requerimientos de energía de las cargas se ajusten a la energía que se puede entregar con equipos ya disponibles, debido a que en el laboratorio se disponía con 4 módulos fotovoltaicos y 2 baterías para la implementación del sistema híbrido. Las características de estos elementos son las siguientes:

MÓDULOS: Schott Solar Solar ASE300-DGF/17 de 12 V nominales

- Potencia nominal: $300 \mathrm{Wp}$

- Tensión de potencia máxima: $17,2 \mathrm{~V}$

- Corriente de potencia máxima: 17,4 A

- Tensión de circuito abierto: $20 \mathrm{~V}$

- Corriente de corto circuito: 19, 1 A

MÓDULOS: BP270F de $12 \mathrm{~V}$ nominales

- Potencia nominal: $70 \mathrm{Wp}$

- Tensión de potencia máxima: $17 \mathrm{~V}$

- Corriente de potencia máxima: 4,1 A 
- Tensión de circuito abierto: $21 \mathrm{~V}$

- Corriente de corto circuito: 4,5 A

BATERÍAS: RWE Schott solar cell

- Capacidad nominal: $200 \mathrm{Ah}$

- Ciclo profundo

- Libre de mantenimiento

- Electrolito de gel

En primera instancia se determina el perfil de carga para establecer la cantidad de horas de uso de cada equipo. Este valor se establece iterativamente hasta el punto de que la cantidad de módulos necesaria para suplir dicha energía, se ajuste a la cantidad de equipos disponibles, pero esto no quiere decir que el equipo de monitoreo se use solo por 6,9 horas al día.

\begin{tabular}{ccccc}
\hline Equipo AC & \multicolumn{5}{c}{$\begin{array}{c}\text { Cantidad Carga Horas de Energía diaria } \\
\text { (W) }\end{array}$} & $\begin{array}{c}\text { uso/día } \\
\text { (W.h/día) }\end{array}$ \\
\hline Equipo de cómputo & 1 & 161,2 & 6,9 & 1112,28 \\
\hline Equipo de adquisición de datos & 1 & 60 & 6,9 & 414 \\
\hline Pantalla & 1 & 22 & 0,3 & 6,6 \\
\hline Equipo DC & Cantidad Carga & Horas de & Energía diaria \\
& & (W) & uso/día & (W.h/día) \\
\hline Nevera & 1 & - & - & 208 \\
\hline
\end{tabular}

Cuadro 1. Perfil de carga considerado.

Seguidamente, se fijan los requerimientos energéticos que debe suplir el sistema.

\begin{tabular}{lc}
\hline Energía AC total diaria (W.h/día) & 1532,880 \\
\hline Energía DC (W.h/día) & 208 \\
\hline Factor Inversor & 0,9 \\
\hline Energía AC corregida (W.h/día) & 1703,200 \\
\hline Energía Total (W.h/día) & 1911,200 \\
\hline Tensión del Sistema (V) & 12 \\
\hline HSS & 4
\end{tabular}

Cuadro 2. Energía que debe suministrarse a las cargas. 
A continuación se define la cantidad de módulos en serie que se ajustan a la tensión nominal establecida para el sistema.

\begin{tabular}{l}
\hline Tensión sistema (V) 12 \\
\hline Tensión módulo (V) 12 \\
\hline \# Módulos en serie 1
\end{tabular}

Cuadro 3. Cantidad de módulos en serie.

Se encuentra la corriente pico del generador y la indicación de la cantidad de módulos fotovoltaicos necesarios para atender dicha carga, para así evaluar que la cantidad calculada corresponde a la cantidad disponible.

\begin{tabular}{lc}
\hline Factor de seguridad & 0,1 \\
\hline Energía total corregida (W.h/día) & 2102,320 \\
\hline$C_{i}$, carga equivalente en corriente (A.h/día) & 175,193 \\
\hline Ipg, intensidad pico del generador (A) & 43,798 \\
\hline Im, corriente de punto máximo (A) & 17,4 \\
\hline Cantidad de módulos en paralelo & 2,517 \\
\hline
\end{tabular}

Cuadro 4. Cantidad de módulos en paralelo.

Ya que la corriente pico de los módulos de baja potencia corresponde a un $25 \%$ de la corriente pico de los módulos de alta potencia, el tener 2 módulos de baja potencia corresponde al $50 \%$ de un módulo de alta potencia, razón por la cual los requerimientos energéticos llevan a que la cantidad óptima de los módulos es 2, 5 , lo que se ajusta a la cantidad de módulos disponibles.

A continuación se establece el dimensionamiento del banco de baterías, donde al igual que se realizó con los módulos fotovoltaicos, se define la cantidad de baterías en serie.

\begin{tabular}{c}
\hline Tensión del sistema (V) 12 \\
\hline Tensión de las baterías (V) 12 \\
\hline \# Baterías en serie 1 \\
\hline
\end{tabular}

Cuadro 5. Cantidad de baterías en serie. 
Posteriormente, se encuentra la cantidad de baterías en paralelo para atender la carga dispuesta para el sistema.

\begin{tabular}{lc}
\hline Días de autonomía & 1,9 \\
\hline$C_{i}$, carga equivalente en corriente (A.h/día) & 175,193 \\
\hline Capacidad nominal del banco de baterías (A.h) & 332,867 \\
\hline Profundidad de descarga & 0,8 \\
\hline Capacidad nominal del banco corregida (A.h) & 416,084 \\
\hline Capacidad nominal de la batería (A.h) & 200 \\
\hline Cantidad de baterías en paralelo & 2,080 \\
\hline
\end{tabular}

Cuadro 6. Cantidad de baterías en serie.

Como vemos, con la disponibilidad de 2 módulos de alta potencia, 2 módulos de baja y 2 baterías se puede suministrar una energía de 2.102 W.h diariamente, y se tienen 1,9 días de autonomía.

En SFV autónomos, se establece que los días de autonomía de un sistema fotovoltaico corresponden a la cantidad de días que las baterías son capaces de alimentar la carga bajo una radiación solar mínima o nula. Para el presente caso de SFV híbrido, dado que, en condición de falla, el suministro de energía que se entrega al sistema de monitoreo y a la nevera debe ser continuo, el concepto de días de autonomía cambia su connotación: las cargas no estarán en operación por intervalos de tiempo como se indico en la tabla de perfil de carga, por lo que el tiempo que el sistema es capaz de realizar un suministro de energía continuo hacia dichas cargas críticas no son los días de autonomía encontrados, sino que corresponde al producto de las horas de uso diario por la cantidad de días de autonomía, encontrándose que bajo las condiciones a las cuales está sujeto el sistema, se puede entregar energía de forma ininterrumpida por 13 horas con radiación solar nula.

\section{Sistema de control del sistema híbrido}

\subsection{Adquisición de datos}

La evaluación del estado del sistema se realizará a partir de la adquisición del nivel de tensión del banco de baterías para evaluar su estado de carga, y la adquisición del nivel de tensión en la red para determinar si este es suficiente para alimentar la carga crítica del sistema. Dichas señales son adquiridas mediante la tarjeta de adquisición de datos NI-USB800 a través de sus canales análogos.

\subsection{Activación de los relés de interconexión}

Según la evaluación de las señales de tensión adquiridas, la tarjeta de adquisición emite señales de activación para los relés de la caja de maniobra a través de 
sus canales digitales. Debido a que dichas señales emitidas tiene una amplitud 5 Vdc con un máximo de $5 \mathrm{~mA}$, se necesitó desarrollar un circuito amplificador de potencia para acondicionar las señales emitidas por la tarjeta a los niveles de tensión y corriente adecuados para excitar las bobinas de los relés. A continuación se muestra el circuito implementado.

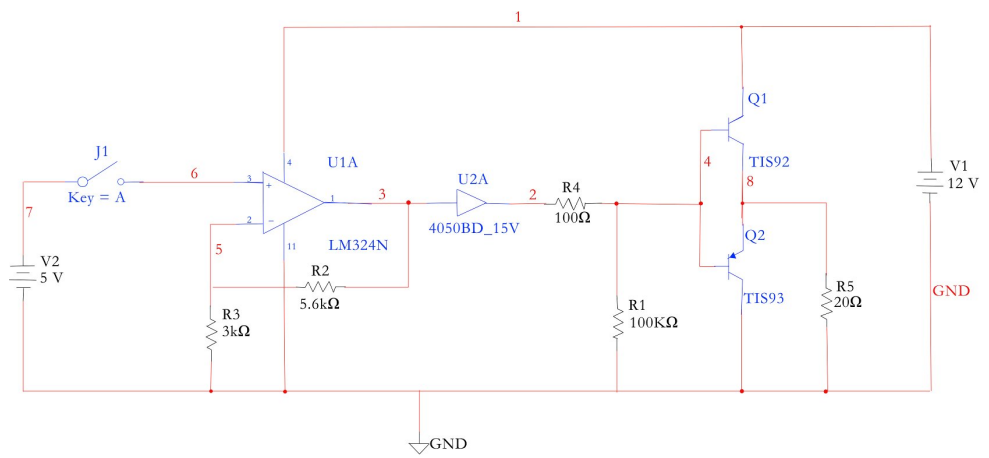

Figura 2. Circuito amplificador de potencia.

\subsection{Regulador de carga}

Para el desarrollo del mismo se utilizó el lenguaje de programación LabVIEW. Este permite realizar programas (llamados instrumentos virtuales, VI) en forma gráfica con los cuales es posible medir señales, hacer adquisición y procesamiento de datos y además control de equipos y procesos. En contraste con los lenguajes de programación basados en texto, donde los instrumentos determinan la ejecución del programa, en LabVIEW, el flujo de datos determina dicha ejecución.

Interfaces del regulador. Una primera parte muestra las señales adquiridas así como los valores característicos de las mismas. Para la señal de la red, se muestra su valor RMS, los valores pico positivo y negativo; para la señal del banco de baterías se muestra su valor de voltaje, así como un indicador tipo tanque para mostrar el estado de carga de las mismas.

La parte restante muestra el esquema del sistema híbrido desarrollado, así como indicadores lumínicos que denotan el estado de los relés de interconexión. Esta interfaz también posee controles manuales de activación de los relés para considerar fallas o mantenimiento de los elementos del sistema, pero por seguridad del sistema, la activación de dichos controles solo tiene efecto si se introduce la adecuada clave de activación. 


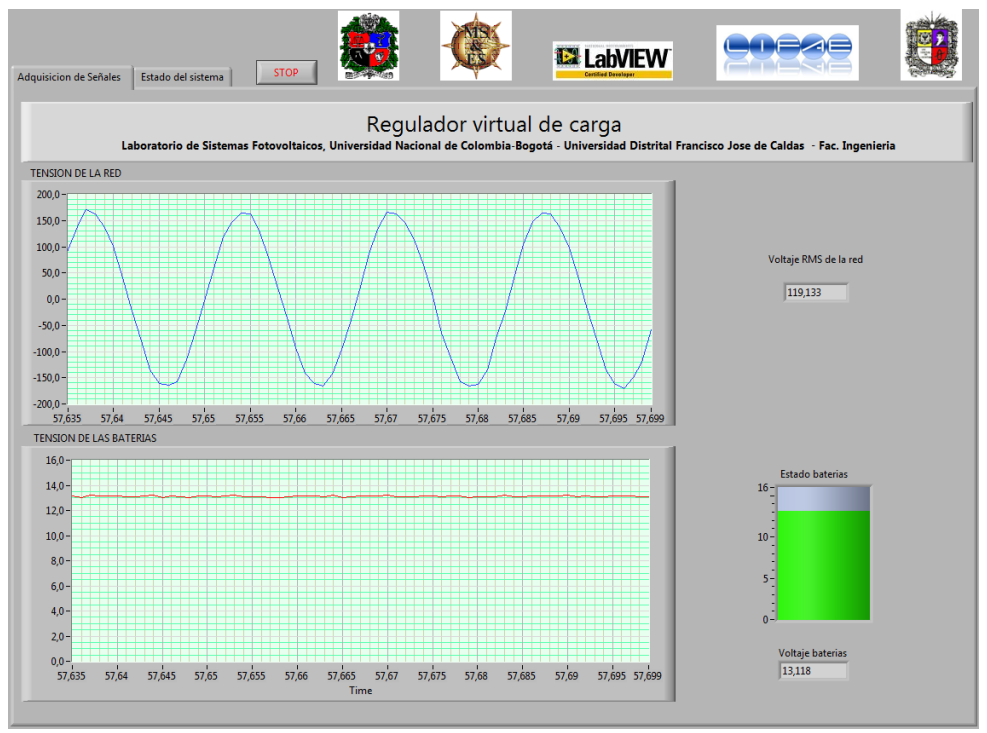

Figura 3. Señales adquiridas por el regulador.

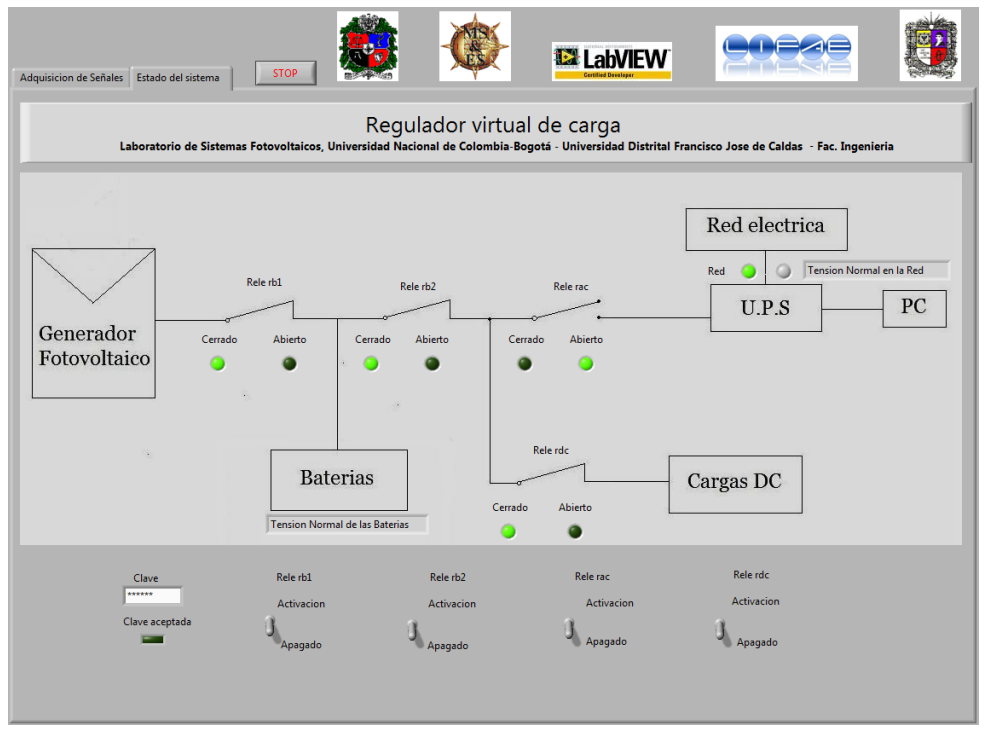

Figura 4. Revisión del estado del sistema híbrido. 
Subsistemas del regulador de carga. A continuación se presentan los diferentes bloques funcionales que definen la operación el regulador virtual de carga desarrollado.

Clave de autorización. Para realizar modificaciones en el sistema o llevar a cabo operaciones manuales, en el panel frontal se debe ingresar una secuencia alfanumérica que coincida con otra secuencia definida como clave de administrador definida en el sistema.

Evaluación de la señal de la red. A la señal sinusoidal de la red se calcula el valor RMS y se compara dicho valor con constantes que indican una ausencia de tensión o sobretensión en la red, donde el valor de dichas constantes depende de los valores límite de la regulación de tensión establecidos por el ente regulador local. Además, la emisión de señales de activación de los relés depende de que el valor RMS de la señal medida de la red se encuentre dentro del rango de valores límite de la regulación de tensión.

Evaluación de la tensión del banco de baterías. En este aspecto, se consideran los estados de sobrecarga o descarga de las mismas. Para la condición de sobrecarga, la comparación del nivel de tensión se realiza respecto al valor que establezca el fabricante como sobrecarga de las baterías, y se emite una señal de activación de relés si la tensión de las baterías es igual o superior al valor establecido como sobrecarga. Para la condición de descarga se establece el valor de tensión que provee el fabricante: emitir una señal de activación hacia los relés si la tensión de las baterías es menor o igual al voltaje al cual se consideran descargadas las baterías.

Evaluación del estado del sistema y activación manual. Para evitar que el sistema guarde los valores medidos de tensión en red y baterías cada vez que realice una iteración, los valores adquiridos se almacenan; y posterormente se realiza una comparación entre el dato actual y el dato evaluado en la iteración anterior, permitiendo la escritura de datos y generación de señales de activación solo cuando ocurran cambios en el sistema.

Para la operación manual del sistema, se configura de manera que la señal a emitirse hacia la etapa de potencia provenga tanto de la evaluación de los valores de tensión del sistema como de la señal generada por los mandos manuales dispuestos.

Escritura de datos según medición de tensión. Cuando el sistema registra que los valores de tensión incumplen las constantes de comparación establecidas anteriormente tanto para la tensión de la red como para la tensión de las baterías, se habilita la escritura de datos y según la magnitud de tensión y el tipo de evento ocurrido, el valor de tensión medido se guarda junto con la descripción del evento. 
Para finiquitar el proceso de escritura de datos, se hace un estampado de tiempo a cada dato guardado a fin de realizar un seguimiento cronológico al comportamiento del sistema. La siguiente figura presenta el formato bajo el cual se genera el archivo de históricos, mostrando como ejemplo el registro efectuado simulando diferentes magnitudes de las señales adquiridas.

\begin{tabular}{|c|c|c|c|c|}
\hline 4 & A & B & C & D \\
\hline 1 & Fecha--13/02/2010--04:31 p.m. & Ausencia de Tension en la red & 104,9733 & Voltios RMS \\
\hline 2 & Fecha--13/02/2010--04:31 p.m. & Conexion a la red de la UPS & 119,6429 & Voltios RMS \\
\hline 3 & Fecha--13/02/2010--04:32 p.m. & Sobretension en la red & 130,2082 & Vo \\
\hline 4 & Fecha--13/02/2010--04:32 p.m. & Tension de la red normal & 125,3305 & Voltios RMS \\
\hline 5 & Fecha--13/02/2010--04:32 p.m. & Descarga de las baterias & 10,4344 & Voltios DC \\
\hline 6 & Fecha--13/02/2010--04:33 p.m. & Reconexion de las & 11,6307 & s DC \\
\hline 7 & Fecha--13/02/2010--04:33 p.m. & Sobrecarga de las baterias & 14,8 & Volt \\
\hline 8 & Fecha--13/02/2010--04:33 p.m. & Reconexion de los modulos & 11,8861 & Voltios DC \\
\hline 9 & Fecha--13/02/2010--04:33 p.m. & Ausencia de Tension en la red & 101,6427 & Voltios RMS \\
\hline 10 & Fecha--13/02/2010--04:33 p.m. & Conexion a la red de la UPS & 109,9339 & Vo \\
\hline 11 & Fecha--13/02/2010--04:33 p.m. & Sobretensi & 134,7 & s RMS \\
\hline 12 & Fecha--13/02/2010--04:34 p.m. & Tension de la red normal & 125,1355 & Voltios RMS \\
\hline 13 & Fecha--13/02/2010--04:34 p.m. & Descarga de las baterias & 9,8755 & OS DC \\
\hline 14 & Fecha--13/02/2010--04:34 p.m. & Reconexion de las cargas DC & 11,9339 & Voltios DC \\
\hline 15 & Fecha--13/02/2010--04:34 p.m. & Sobrecarga de las baterias & 14,7947 & Voltios DC \\
\hline
\end{tabular}

Figura 5. Formato de registro de eventos del sistema.

\section{Pruebas y resultados}

Tras haber realizado el acople entre el sistema fotovoltaico y la UPS, se realizó intencionalmente un corte al suministro de energía a la UPS y se encontró que la carga crítica demandaba una corriente entre 20 y $25 \mathrm{Amp}$. Al revisar el aporte de corriente que cada una de las partes del sistema daba a la carga, se encontró que en los primeros instantes en los cuales el sistema detectaba ausencia de tensión de la red, las baterías de la UPS aportaban casi un $65 \%$ de la corriente demandada mientras que las baterías del sistema fotovoltaico aportaban la corriente excedente. Sin embargo, con el paso del tiempo, la batería de la UPS comienza a disminuir el monto de corriente que aporta mientras que la corriente entregada por las baterías aumenta, presentándose esta situación progresivamente conforme aumenta el tiempo de ausencia de tensión en la red, pero sin interrumpir el flujo de corriente hacia el inversor de la UPS para alimentar la carga crítica.

En el momento en que se lleva a cabo el acople del sistema fotovoltaico con la UPS, se realiza un respaldo energético de forma efectiva ya que se aumenta la capacidad de las batería que percibe la UPS para realizar el suministro de energía, además si se presenta la adecuada incidencia de luz solar, la corriente fotogenerada aporta corriente a la carga y al banco de baterías, manteniendo 
constante el nivel de tensión en las mismas y evitando su descarga. En cuanto a la operación del regulador de carga, éste registra efectivamente la ausencia de tensión y emite las señales de activación de los relés para permitir que el sistema fotovoltaico se conecte con la UPS.

El sistema fotovoltaico híbrido completo, se encuentra en correcto y satisfactorio funcionamiento desde mediados de diciembre de 2009.

\section{Conclusiones}

1. Se presenta una aplicación particular de los sistemas fotovoltaicos, en la cual se establece un sistema de transferencia de fuente para alimentar cargas consideradas como críticas, donde la energía generada por los paneles fotovoltaicos se orienta para alimentar cargas DC y mantener cargado un banco de baterías que se acoplará a las baterías contenidas de una UPS, pues con ella se energiza la carga crítica cuando haya ausencia de tensión en la red de suministro eléctrico.

2. Es de tener en cuenta el hecho de que la UPS maneja su referencia de tensión a partir del establecimiento de una tierra flotante, razón por la cual se debe verificar que el sistema fotovoltaico se mantenga con referencia de tensión flotante para poder acoplar la batería embebida en la UPS, ya que de lo contrario se producirán cortocircuitos que dañarán los componentes electrónicos de la UPS.

3. Con la metodología aplicada y el correcto establecimiento de los perfiles de carga que el sistema debe alimentar, se satisfacen adecuadamente los requerimientos energéticos de las cargas con la cantidad de módulos y baterías de los cuales se disponía para el desarrollo del sistema de respaldo.

4. Para el desarrollo de trabajos futuros, se sugiere implementar un dispositivo electrónico que permita regular la corriente con la cual se cargan las baterías, evitando así que se presenten sobrecargas constantes y optimizando el estado de carga de las mismas. Esta situación se presenta debido a que frente alta radiación solar, se presenta una alta corriente fotogenerada que se inyecta a las baterías y resulta en alta pendiente de elevación de tensión en bornes de la batería, siendo este un proceso de carga inadecuado.

\section{Referencias}

1. Bayegan, M.: A Vision of the Future Grid IEEE Power Engineering Review. (2001)

2. Lawrence, R., Middlekauff, S.: The New Guy on the Block IEEE Industry Applications Magazine. January - February (2005)

3. Aristizábal J., Arredondo, C., Hernandez, J., Gordillo, G.: Development of equipment for monitoring PV power plants, using Virtual Instrumentation, 2006 IEEE 4th World Conference on Photovoltaic Energy Conversion, May. 7-12, 2006, Hawaii, U.S.A. (2006)

4. Hernández, J.: Diseño e instalación del primer sistema fotovoltaico interconectado en Colombia y desarrollo de equipo para su monitoreo [Tesis de Maestría], Bogotá, Universidad Nacional de Colombia, Maestría en Ingeniería Eléctrica. (2006) 
5. Markvart, T.: Solar Electricity, John Wiley and Sons, Inc., N.Y. (2000)

6. Aguilar, J., Pérez, P., De la Casa, J.: Calculo de energía generada por un sistema fotovoltaico conectado a la red, Jaén. Universidad de Jaén, Ingeniería Técnica Industrial. (2004)

7. Institute of Electrical and Electronics Engineers (IEEE): Recommended Practice for Utility Interface of Photovoltaic (PV) Systems Std 929, EE.UU., IEEE. (2000),

8. Forero N., Hernández J., Gordillo G.: Development of a Monitoring System for a PV Solar Plant, Energy Conversion and Management. Vol.47 2329-2336, (2006)

9. Dettmer R,: Empowering Distributed Generation IEE Review January (2003)

10. Gasquet, H.: Conversión de la luz solar en Energía Eléctrica: Manual Teórico y Práctico sobre los sistemas Fotovoltaicos. Cuernavaca. Solartronics S.A (2004)

11. Wiles, J.: Photovoltaic Power System and The National Electrical Code: Sugested Practices. Las Cruces, New Mexico. Southwest Technology Development Institute. (1996) 